\title{
Stringent bounds to spatial variations of the electron-to-proton mass ratio in the Milky Way
}

\author{
P. Molaro ${ }^{\text {a }}$, S. A. Levshakov ${ }^{\mathrm{b}}$, and M. G. Kozlov ${ }^{\mathrm{c}}$ \\ a INAF-Osservatorio Astronomico di Trieste, \\ Via G. B. Tiepolo 11, 34143 I, Trieste, Italy \\ bIoffe Physical-Technical Institute, \\ Politekhnicheskaya Str. 26, 194021 St. Petersburg, Russia \\ ${ }^{c}$ Petersburg Nuclear Physics Institute, \\ Gatchina, 188300, Russia
}

The ammonia method, recently proposed by Flambaum and Kozlov (2007) to probe variations of the electronto-proton mass ratio, $\mu=m_{\mathrm{e}} / m_{\mathrm{p}}$, is applied for the first time to dense prestellar molecular clouds in the Milky Way, allowing to test $\Delta \mu / \mu$ at different galactocentric distances. High quality radio-astronomical observations are used to check the presence of possible relative radial velocity offsets between the inversion transition of $\mathrm{NH}_{3}$ $(J, K)=(1,1)$, and the CCS $J_{N}=2_{1}-1_{0}$ and $\mathrm{N}_{2} \mathrm{H}^{+} J=1-0$ rotational transitions. Carefully selected sample of $21 \mathrm{NH}_{3} / \mathrm{CCS}$ pairs observed in the Perseus molecular cloud provide the offset $\Delta V_{\mathrm{CCS}-\mathrm{NH}_{3}}=36 \pm 7$ stat $\pm 13.5_{\text {sys }}$ $\mathrm{m} \mathrm{s}^{-1}$. A similar offset of $\Delta V=40.8 \pm 12.9_{\text {stat }} \mathrm{m} \mathrm{s}^{-1}$ between $\mathrm{NH}_{3}(J, K)=(1,1)$ and $\mathrm{N}_{2} \mathrm{H}^{+} J=1-0$ has been found in an isolated dense core L183 by Pagani et al. (2009).

Overall these observations provide a safe bound of a maximum offset between ammonia and the other molecules at the level of $\Delta V \leq 100 \mathrm{~m} \mathrm{~s}^{-1}$. Being interpreted in terms of $\Delta \mu / \mu$, this bound corresponds to $\Delta \mu / \mu \leq 1 \times 10^{-7}$, which is an order of magnitude more sensitive than available extragalactic constraints. Taken at face value the measured $\Delta V$ shows positive shifts between the line centers of $\mathrm{NH}_{3}$ and these two other molecules and suggest a real offset, which would imply a $\Delta \mu / \mu \sim 4 \times 10^{-8}$. If $\Delta \mu / \mu$ follows the gradient of the local gravitational potential, then the obtained results are in conflict with laboratory atomic clock experiments in the solar system by $\sim 5$ orders of magnitude, thus requiring a chameleon-type scalar field model. New measurements involving other molecules and a wider range of objects along with verification of molecular rest frequencies are currently planned to confirm these first indications.

\section{Introduction}

The late acceleration in the universal expansion reveals a negative-pressure component of the bulk of energy density, 'dark energy'. This can be a cosmological constant or a dynamically evolving scalar field $\varphi$, 'quintessence', which does not have a cosmic coincidence problem. However, in quintessence the coupling of scalar field to ordinary matter leads unavoidably to long-range forces with a variability of the physical constants and a violation of the Equivalence Principle. Effects that are currently searched in laboratory, space-based experiments and astronomical obser- vations.

The suitable constants are the fine-structure constant $\alpha=e^{2} /(\hbar c)$ and the electron-to-proton mass ratio $\mu=m_{\mathrm{e}} / m_{\mathrm{p}}$, or different combinations of them with the proton gyromagnetic ratio $g_{\mathrm{p}}$ [4].

Accurate laboratory atomic clocks experiments constraint the temporal variation of $\alpha$ of $\dot{\alpha} / \alpha=$ $(-1.6 \pm 2.3) \times 10^{-17} \mathrm{yr}^{-1}[19]$. Analysis of quasar absorption-line systems provided $\Delta \alpha / \alpha=$ $(-4.6 \pm 1.1) \times 10^{-6}\left[14\right.$ or $|\Delta \alpha / \alpha| \leq 6 \times 10^{-6}$ 2211. Here $\Delta \alpha / \alpha=\left(\alpha^{\prime}-\alpha\right) / \alpha$, with $\alpha$ denoting the value of the fine-structure constant in the laboratory and $\alpha^{\prime}$ the specific absorp- 


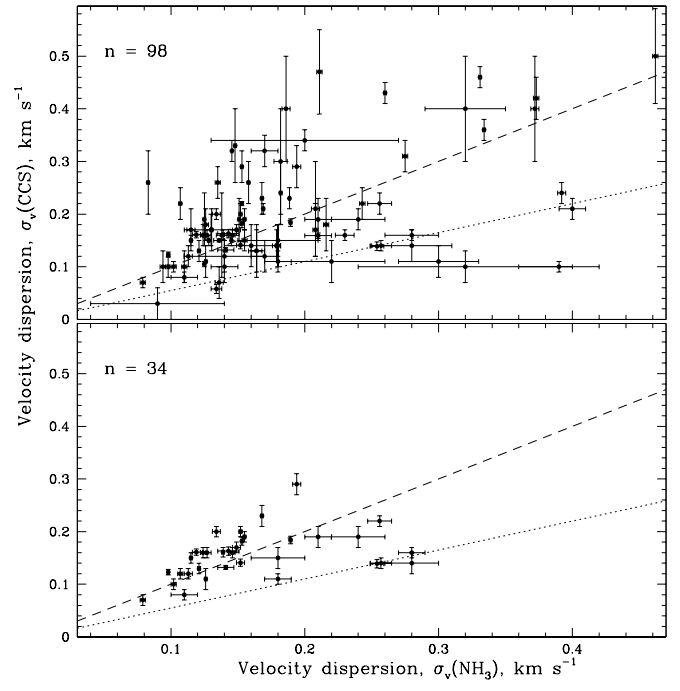

Figure 1. Upper panel: CCS $\left(2_{1}-1_{0}\right)$ versus $\mathrm{NH}_{3}(1,1)$ linewidths for cores in the Perseus molecular cloud from 20 . The error bars represent $1 \sigma$ standard deviations. Lower panel: selected sample with single-component profiles. The dashed and dotted lines are the boundaries for pure turbulent and pure thermal line broadening.

tion/emission line system of a galactic or extragalactic object, the same definition is applied to $\Delta \mu / \mu$. Being linearly extrapolated to redshift $z \sim 2$, or $t \sim 10^{10} \mathrm{yr}$, the laboratory bound leads to $|\Delta \alpha / \alpha| \leq 4 \times 10^{-7}$ which is below the astronomical limits or claims for variability. However, laboratory experiments and quasar absorption spectra probe different time scales and regions of the universe, and the connection between them is somewhat model dependent [12].

The direct laboratory estimate of time variation of $\mu$ gives $\dot{\mu} / \mu=(1.6 \pm 1.7) \times 10^{-15} \mathrm{yr}^{-1}[2$. Astrophysical estimates of changes of $\mu$ at high redshifs are controversial with claims of nonzero

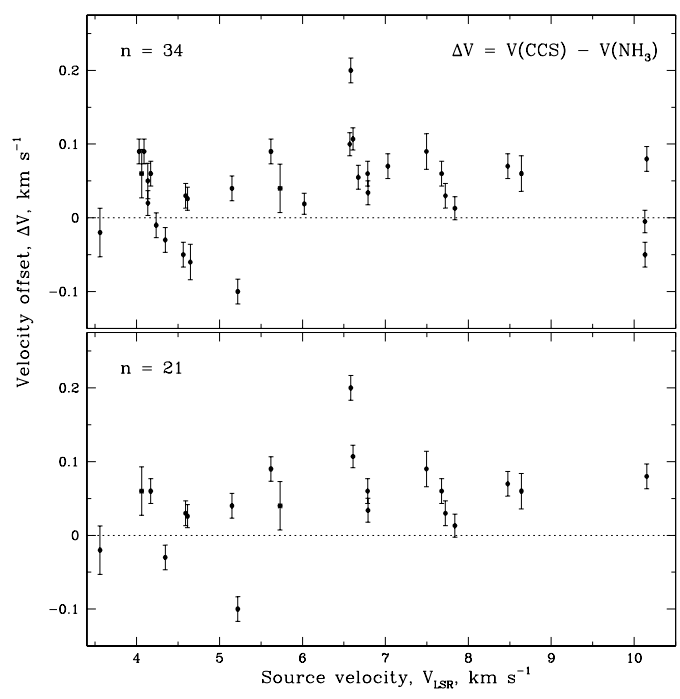

Figure 2. Upper panel: Velocity offset $\Delta V_{\mathrm{CCS}-\mathrm{NH}_{3}}$ versus the radial velocity for points shown in Fig. 1. Lower panel: Same as the upper panel but for the points with consistent broadening between the lines in Fig 1. The vertical error bars include both random and systematic errors caused by the uncertainties of the adopted rest frequencies.

and zero changes: $\Delta \mu / \mu=(-2.4 \pm 0.6) \times 10^{-5}$ was inferred from the analysis of the $\mathrm{H}_{2}$-bearing clouds at $z=2.6$ and 3.0 [17], but this value was not confirmed later [7. More stringent bounds are obtained at redshifts $z=0.68$ and $z=0.89$ from the analysis of radio-frequency transitions in $\mathrm{NH}_{3}$ and other molecules such as $\mathrm{CO}, \mathrm{HCO}^{+}$, $\mathrm{HC}_{3} \mathrm{~N}$ providing $\Delta \mu / \mu=(-0.6 \pm 1.9) \times 10^{-6}$, $\Delta \mu / \mu=(0.08 \pm 0.47) \times 10^{-6}$ [45], which favors a non-variability of $\mu$ at a level of $\sim 1.5 \times 10^{-6}$.

In these studies it is implicitly assumed that the rate of time variations dominates over possible spatial variations. However, if scalar fields trace the gravitational field inhomogeneities spa- 
tial variation could be present as well 3. The circumstance of spatial variations of constants are tested by measurements of the atomic clock transition in laboratories on Earth orbiting in the changing gravitational potential of the Sun. The laboratory experiments constrain the couplings of $\alpha$ and $\mu$ to the gravitational field at a level of $k_{\alpha, \mu} \sim 10^{-6}, 10^{-5}$ [2]. To reconcile these results with quintessence, chameleon-type models were suggested which allow scalar fields to evolve on cosmological time scales today and to have simultaneous strong couplings of order unity to matter [8]. In these models the mass of the scalar field depends on the local matter density, which explains why cosmological scalar fields, such as quintessence, are not detectable in local tests of the Equivalence Principle. On Earth in a dense environment the mass of the field can be sufficiently large and $\varphi$-mediated interactions are short-ranged. On the other hand, the cosmological matter density is about $10^{30}$ times smaller and the mass of the scalar field can be very low, of order of $H_{0}$, allowing the field to evolve cosmologically.

In this paper we apply the ammonia method for the first time to Milky Way sources. A detailed and exaustive account has been provided in [10].

\section{Interstellar molecules probe $\Delta \mu / \mu$ spa- tial variations}

Among molecules, ammonia is of particular interest due to its high sensitivity to changes in the electron-to-proton mass ratio, $\mu$ [4] For $\mathrm{NH}_{3}$, the sensitivity coefficient of the inversion transition $\nu=23.69 \mathrm{GHz}$ was calculated in [4]:

$\frac{\Delta \nu}{\nu}=4.46 \frac{\Delta \mu}{\mu}$.

By comparing the observed inversion frequency of $\mathrm{NH}_{3}(1,1)$ with a suitable rotational frequency of another molecule arising co-spatially, a limit on the spatial variation of $\mu$ can be determined:

$\frac{\Delta \mu}{\mu}=0.289 \frac{V_{\mathrm{rot}}-V_{\mathrm{inv}}}{c} \equiv 0.289 \frac{\Delta V}{c}$,

where $V_{\text {rot }}$ and $V_{\text {inv }}$ are the apparent radial velocities of the rotational and inversion transitions, respectively.
Thus the comparison of the relative radial velocities of ammonia inversion lines and rotational transitions of another N-bearing $\left(\mathrm{N}_{2} \mathrm{H}^{+}\right)$and Cbearing $\left(\mathrm{CCS}, \mathrm{HC}_{3} \mathrm{~N}\right)$ molecules can be used to set a limit on spatial variations of $\Delta \mu / \mu$. These molecules are often detected in the molecular clouds allowing to probe this constant in the Milky Way. In molecular clouds considerable fraction of material is not participating in the process of star formation residing in cold and dense clouds which are well suited for accurate measurements. These can be low-mass protostellar cores containing infrared sources, or even starless cores without embedded luminous sources. The physical conditions of the latter are of dense molecular gas preceding gravitational collapse and when the gas density in the core center is $n_{\mathrm{H}_{2}} \leq 10^{5} \mathrm{~cm}^{-3}$ they can be dynamically stable against gravitational contraction [6]. Typical cores have mean gas density $n_{\mathrm{H}} \sim(1-2) \times 10^{4}$ $\mathrm{cm}^{-3}$, velocity dispersion $\sigma_{v}=0.17 \mathrm{~km} \mathrm{~s}^{-1}$, kinetic temperature $T_{\text {kin }}=11 \mathrm{~K}$, radius $R=0.09$ pc, mass $\mathcal{M} \sim 1 \mathcal{M}_{\odot}$ and $\mathrm{NH}_{3}, \mathrm{~N}_{2} \mathrm{H}^{+}, \mathrm{CCS}$, and $\mathrm{HC}_{3} \mathrm{~N}$ are usually observed in emission.

\section{Cores in the Perseus molecular cloud}

An ammonia spectral atlas of 193 dense protostellar and prestellar cores of low masses in the Perseus molecular cloud is provided by 20 . The spectral observations of the cores in $\mathrm{NH}_{3}$ $(1,1),(2,2), \operatorname{CCS}\left(2_{1}-1_{0}\right)$ and $\mathrm{CC}^{34} \mathrm{~S}\left(2_{1}-1_{0}\right)$ lines were carried out simultaneously using the 100-m Green Bank Telescope (GBT). Each target was observed in a single-pointing, frequencyswitched mode. The GBT beam size at $23 \mathrm{GHz}$ is $F W H M=31^{\prime \prime}$ or $0.04 \mathrm{pc}$ at the distance of 260 pc of Perseus cloud. The spectral resolution was $24 \mathrm{~m} \mathrm{~s}^{-1}$ and both molecules were observed with similar angular and spectral resolutions.

The atlas of 193 sources is very conveniently presented on the website (see 20] for details) where the original spectra are shown along with the best fitting models, model parameters and their uncertainties. The number of cores where both CCS $\left(2_{1}-1_{0}\right)$ and $\mathrm{NH}_{3}(1,1)$ lines were detected is 98 . The central velocities of these lines, being averaged with weights inversely pro- 
portional to the variances of the measurements, reveal that $\Delta V_{\mathrm{CCS}-\mathrm{NH}_{3}}=16 \mathrm{~m} \mathrm{~s}^{-1}$. It was suggested [20] that this offset is due to uncertainties in the assumed rest frequency of the CCS line which is of $13.5 \mathrm{~m} \mathrm{~s}^{-1}$ (cfr Table 1). However, the unweighted averaging, which is more appropriate in the presence of unaccounted errors, gives $\Delta V_{n=98}=44 \pm 13 \mathrm{~m} \mathrm{~s}^{-1}$

Many cores show evidence for the multiple velocity components along the line-of-sight. The selection of simple profiles which have been satisfactorily fitted by a single component model provides a sample of $n=34$ pairs The weighted mean for this sample gives $\Delta V_{n=34}^{w}=40 \pm 10 \mathrm{~m} \mathrm{~s}^{-1}$. and $\Delta V_{n=34}=39 \pm 10 \mathrm{~m} \mathrm{~s}^{-1}$ for the unweighted mean 10 .

A further refinement of the sample could be done taking only cores which show line broadening values between pure turbulent, $\sigma_{v}(\mathrm{CCS})=$ $\sigma_{v}\left(\mathrm{NH}_{3}\right)$, and pure thermal, $\sigma_{v}(\mathrm{CCS})=$ $0.55 \sigma_{v}\left(\mathrm{NH}_{3}\right)$, regimes, which should be the case if both emission lines arise co-spatially. This requirement further reduce the sample to $n=21$ clouds with $\Delta V_{n=21}^{w}=50 \pm 0.013 \mathrm{~m} \mathrm{~s}^{-1}$, and $\Delta V_{n=21}=48 \pm 13 \mathrm{~m} \mathrm{~s}^{-1}$ for the weighted and unweighted mean respectively.

The fact that scatter remains rather constant in the various samples reflects the complexity of gas kinematics and effects of chemical segregation of one molecule with respect to the other in dense molecular cores which are the dominant sources of error.

The unweighted mean from the $n=21$ sample could be taken as the best estimate of the velocity offset for the Perseus dark cores, but in fact we could have taken any of the former samples without changing significantly the result. When we account for uncertainties in the rest frequencies, we have $\Delta V_{\mathrm{CCS}-\mathrm{NH}_{3}}=48 \pm 13 \pm 13.5$ $\mathrm{m} \mathrm{s}^{-1}$. Other observations in the Pipe nebula [18, and in Infrared dark clouds [21] are examined in [10. 8 pairs in the Pipe Nebula provide $\Delta V_{\mathrm{CCS}-\mathrm{NH}_{3}}=53 \pm 11_{\text {stat }} \pm 13.5_{\text {sys }}$ $\mathrm{m} \mathrm{s}^{-1} .36 \mathrm{NH}_{3} / \mathrm{N}_{2} \mathrm{H}^{+}$and $27 \mathrm{NH}_{3} / \mathrm{HC}_{3} \mathrm{~N}$ pairs observed in Infrared Dark Clouds, which are much more massive than the dense cores, provide $\Delta V_{\mathrm{N}_{2} \mathrm{H}^{+}-\mathrm{NH}_{3}}=148 \pm 32_{\text {stat }} \pm 13.6_{\text {sys }} \mathrm{m} \mathrm{s}^{-1}$ and $\Delta V_{\mathrm{HC}_{3} \mathrm{~N}-\mathrm{NH}_{3}}=115 \pm 37_{\text {stat }} \pm 31_{\text {sys }} \mathrm{m} \mathrm{s}^{-1}$, respec- tively, thus supporting the results found in the Perseus region.

\section{The L183 dense core}

Recently Pagani et al. [16] have compared the $\mathrm{N}_{2} \mathrm{H}^{+}$and $\mathrm{N}_{2} \mathrm{D}^{+}$transitions with the transitions of $\mathrm{NH}_{3}$ in the dark cloud L183. The whole elongated dense core of L183 has been fully mapped in $\mathrm{N}_{2} \mathrm{H}^{+}$and $\mathrm{N}_{2} \mathrm{D}^{+}(\mathrm{J}: 1-0)$ lines with the IRAM 30$\mathrm{m}$ telescope with velocity resolution in the range $30-50 \mathrm{~m} \mathrm{~s}^{-1}$. Spatial resolution ranges from 33 arcsec at $77 \mathrm{GHz}$ to 9 arcsec at $279 \mathrm{GHz}$. The spatial sampling is 12 arcsec for the main prestellar core and 15 arcsec for the southern and northern extension of the prestellar core. Observations of $\mathrm{NH}_{3}(1,1)$ and $(2,2)$ inversion lines have been performed at the Green Bank 100-m telescope with velocity sampling of $20 \mathrm{~m} \mathrm{~s}^{-1}$, angular resolution of $\sim 35$ arcsec and spatial sampling of $24 \operatorname{arcsec}$ all over the source.

Extremely narrow molecular lines observed in this cold dark cloud provide a sensitive spectroscopic tool to track small systematic velocity gradients and to align values of poorly known frequencies with more reliable ones. For the latter, $\mathrm{NH}_{3}$ is the molecule of choice since it is stable and its frequencies are accurately measured in the laboratory. $\mathrm{NH}_{3}(1,1)$ average frequency from the whole HFS is $\nu=23694495487( \pm 48) \mathrm{Hz}$ [9], or $\nu=23694495481 \pm 22 \mathrm{~Hz}$ from the revision of Hougen [13]. Thus $\mathrm{NH}_{3}$ provides a reference transition known with a precision of few $10^{-10}$ or $\sim 0.6 \mathrm{~m} \mathrm{~s}^{-1}$.

Ammonia and diazenylium have the same chemical origin, starting from $\mathrm{N}_{2}$ and are wellknown to be coexistent as discussed by e.g. 2324. In L183 the velocity along the dense filament is constantly changing suggesting a flow towards the prestellar cores and a rotation of the filament around its vertical axis. $\mathrm{NH}_{3}(1,1), \mathrm{N}_{2} \mathrm{H}^{+}$ and $\mathrm{N}_{2} \mathrm{D}^{+}(\mathrm{J}: 1-0)$ all trace exactly the same gradients and it seems therefore compulsory that their velocities be identical as there is no obvious possibility that the velocity gradients be exactly parallel but offset from each other, especially in the case of a cylinder rotation. Finally, the fact that $\mathrm{N}_{2} \mathrm{D}^{+}$velocity centroids are almost identi- 
Table 1

Molecular transitions and uncertainties, $\varepsilon_{v}$, The numbers in parentheses correspond to $1 \sigma$ errors.

\begin{tabular}{llr}
\multicolumn{1}{c}{ Transition } & \multicolumn{1}{c}{$\begin{array}{c}\nu_{\text {rest }}, \\
\mathrm{GHz}\end{array}$} & $\begin{array}{r}\varepsilon_{v}, \\
\mathrm{~m} \mathrm{~s}^{-1}\end{array}$ \\
\hline $\mathrm{CCS} J_{N}=2_{1}-1_{0}$ & $22.344033(1)$ & 13.4 \\
$\mathrm{NH}_{3}(J, K)=(1,1)$ & $23.694495481(22)$ & 0.6 \\
$\mathrm{HC}_{3} \mathrm{~N} J=5-4$ & $45.4903102(3)$ & 2.8 \\
$\mathrm{~N}_{2} \mathrm{H}^{+} J=1-0$ & $93.173777(4)$ & 13.5
\end{tabular}

cal with those of $\mathrm{N}_{2} \mathrm{H}^{+}$indicates that the different opacities of the lines are not introducing any measurable bias here.

A similar behaviour of all the three molecules shows that these share the same volume of the cloud and undergo the same macroscopic velocity shifts.

On the basis that all the three species are spatially coexistent and trace the same velocities Pagani et al 16] used the observed offset to adjust the frequencies of $\mathrm{N}_{2} \mathrm{H}^{+}$and $\mathrm{N}_{2} \mathrm{D}^{+}$to that of $\mathrm{NH}_{3}$. For the reference position alone, the difference is of $\Delta V_{\mathrm{N}_{2} \mathrm{H}^{+}-\mathrm{NH}_{3}}=40.8 \pm 0.56 \mathrm{~m} \mathrm{~s}^{-1}$ and $\Delta V_{\mathrm{N}_{2} \mathrm{H}^{+}-\mathrm{NH}_{3}}=40.8 \pm 12.9 \mathrm{~m} \mathrm{~s}^{-1}$ on 65 common positions . For the three transitions of $\mathrm{N}_{2} \mathrm{D}^{+}$, the direct comparison of the reference position with $\mathrm{NH}_{3}(1,1)$ spectrum yields $(\mathrm{J}: 1-0): 37.7 \pm 0.85$ $\mathrm{m} \mathrm{s}^{-1}(\mathrm{~J}: 2-1): 47.7 \pm 0.92 \mathrm{~m} \mathrm{~s}^{-1}(\mathrm{~J}: 3-2): 63.6 \pm$ $4.7 \mathrm{~m} \mathrm{~s}^{-1}$ and similar offsets are found for $\mathrm{N}_{2} \mathrm{D}^{+}$ (cfr [16] for details).

The rest frequencies for the molecule $\mathrm{N}_{2} \mathrm{H}^{+}$in [16] analysis were taken from [1] and are known with precision of $\sim 7 \mathrm{kHz}$. However, an error of $4 \mathrm{KHz}$ is given in the Cologne Database for Molecular Spectroscopy (CDMS). The difference between the data from [1] and the CDMS frequencies is $-12 \mathrm{~m} \mathrm{~s}^{-1}$ and the Pagani et al velocity offset between $\mathrm{N}_{2} \mathrm{H}^{+}$and $\mathrm{NH}_{3}$ should be corrected by this difference when adopting the CDMS rest frequency.

\section{Discussion}

\subsection{A safe bound for $\Delta \mu / \mu$}

At the high spectral resolution of these observations the errors in the molecular line posi- tion measurements are mainly restricted by the uncertainties in laboratory frequencies, $\varepsilon_{\nu} \sim 1$ $\mathrm{kHz}$, which correspond to the $V_{\mathrm{LSR}}$ uncertainties of $\varepsilon_{v} \sim 10 \mathrm{~m} \mathrm{~s}^{-1}$ Taking into account that $\Delta \mu / \mu \sim 0.3 \Delta V / c$, these errors implies a sensitivity in $\Delta \mu / \mu$ at a level of $\sim 10^{-8}$, i.e. about 100 times more sensitive than the $\Delta \mu / \mu$ estimate deduced at $z=0.68$ [4]. This level of accuracy can be achieved when assuming that molecules are co-spatially distributed within the cloud, and are observed simultaneously with the same receiver, beam size, system temperature, and velocity resolution. Violation of any of these conditions leads to shifts of the line centers. Overall the observations in the Perseus dense cores as well as in the L183 core allow a maximum offset between ammonia and the other molecules of $\Delta V \leq 100$ $\mathrm{m} \mathrm{s}^{-1}$ in the most conservative way, which corresponds to $\Delta \mu / \mu \leq 1 \times 10^{-7}$, i.e. an order of magnitude more sensitive than previous astronomical constraints on $\mu$.

This firm bound is in conflict with the value $\Delta \mu / \mu=(-24 \pm 6) \times 10^{-6}$ obtained from molecular hydrogen $\mathrm{H}_{2}$ absorption lines at $z \sim 3[17$. However, the variability of $\mu$ was not confirmed at the level of $|\Delta \mu / \mu| \leq 4.9 \times 10^{-5}[25]$ and, recently, a more stringent limit on $\Delta \mu / \mu$ was found at $z \sim 3, \Delta \mu / \mu=(2.6 \pm 3.0) \times 10^{-6}[7]$.

These extragalactic measurements of $\Delta \mu / \mu$ are performed in systems in which gas densities are similar to those in the Milky Way clouds. Thus also in the high- $z$ absorbers the dependence of $\mu$ on $\rho$ is extremely weak, and the value of $\Delta \mu / \mu$ in quasar absorbers is expected to be at the same level as in the interstellar clouds, i.e. $\sim 10^{-7}$. If a $\Delta \mu / \mu$ is found above this value, then the variation should be temporal rather than spatial.

\subsection{Hints of variation of $\Delta \mu / \mu$ ?}

Taken at face value the measured $\Delta V$ in Perseus and L183 being similar while involving different molecules show the possibility of a positive shifts between the line centers of $\mathrm{NH}_{3}$ and other molecules of the order of $40 \mathrm{~m} \mathrm{~s}^{-1}$. This would imply a $\Delta \mu / \mu \sim 4 \times 10^{-8}$.

In theories which consider a direct coupling between the local matter density and the scalar fields driving varying constants the spatial change 
of the constants is proportional to the local gravitational potential [15] (and references cited therein). The effect of scalar fields on $\mu$ can be expressed as

$$
\frac{\Delta \mu}{\mu}=k_{\mu} \Delta \Phi,
$$

where $k_{\mu}$ is a dimensionless coupling constant of a very light scalar field to the local gravitational potential $\Phi$, and $\Delta \Phi$ is the difference of the gravitational potentials between two measurement points. Since in our case $\Delta \Phi \sim 10^{-7}$, a value of $\Delta \mu / \mu=4 \times 10^{-8}$ would require $k_{\mu} \sim 1$, which is inconsistent with the $k_{\mu} \leq 10^{-5}$ estimated from atomic clock experiments [2] .

However, chameleon models are specifically conceived to circumvent this type of conflict. The baryon masses and coupling constants are strongly dependent on the local matter density, $\rho$, 15. The dynamics of the scalar field in these models depends on $\rho$ : in low-density environments it is determined by the scalar field potential $V(\varphi)$, whereas in high-density regions like the Earth's surface it is set by the matter- $\varphi$ coupling. The range of the scalar-mediated force for the terrestrial matter densities is then very short, less than $1 \mathrm{~mm}$ [15. Because of the strong $\varphi$-coupling to matter the measurements of the frequency drifts in the atomic clock experiments ought to be insensitive to the changes in the gravitational potential at Earth caused by the eccentricity of Earth's orbit.

We note that the difference of matter densities between the terrestrial environment $\left(\rho_{\oplus} \sim\right.$ $\left.3 \times 10^{24} \mathrm{GeV} \mathrm{cm}^{-3}\right)$ and, e.g., dense molecular cores $\left(\rho_{\text {cloud }} \sim 3 \times 10^{5} \mathrm{GeV} \mathrm{cm}^{-3}\right)$ is of 20 orders of magnitude. Thus, $\Delta \mu / \mu$ variation $\Delta \mu / \mu=4 \times 10^{-8}$ require models which treat the $\varphi$-mediated force as a short-range force depending on the matter density. In accord with model predictions, the estimated value of the scalar field is much less than one. In this way the measured spatial variation of $\Delta \mu / \mu$ does not contradict the laboratory studies on atomic clocks due to the extremely different density environment in the terrestrial measurements and in the interstellar medium.

\section{Conclusions}

1. Several molecules and in different environments in the Milky Way are used to probe $\Delta \mu / \mu$ variations by means of the $\mathrm{NH}_{3}$ method. This is done by using CCS measured in almost one hundred of dense Perseus clouds and $\mathrm{N}_{2} \mathrm{H}^{+}$and $\mathrm{N}_{2} \mathrm{D}^{+}$in a detailed high spatial resolution survey of the dense core L183.

2. These observations provide evidence that $\mathrm{NH}_{3}$ does not change by more than 100 $\mathrm{m} \mathrm{s}^{-1}$ allowing us to place a robust bound of $\Delta \mu / \mu \leq 10^{-7}$ which is more than an order of magnitude more stringent than the extragalactic bounds of the same quantity.

3. Both sets of comparison point for a common offset of about $40 \mathrm{~m} \mathrm{~s}^{-1}$. Although it is possible that this offset results from rest frequency uncertainties the fact that several transitions are involved suggest that the effect might be real. An intrinsic shift of $40 \mathrm{~m} \mathrm{~s}^{-1}$ would correspond to $\Delta \mu / \mu \sim$ $4 \times 10^{-8}$.

4. To cope with solar system experiments the effect would require chameleon-type scalar field models which predict a strong dependence of masses and coupling constants on the ambient matter density.

5. New observations to verify this possibility are solicited, and some are underway at radio telescopes of Medicina, Effelsberg and Nobeyama.

Acknowledgments The authors are grateful to Eric Rosolowsky, Takeshi Sakai, and Jill Rathborne who sent us additional comments on their observations and data reduction. We thank Paola Caselli, Irina Agafonova, Keith Olive, Alexander Lapinov and Thomas Dent for useful comments and discussions. SAL is supported by the RFBR grant 09-02-00352-a.

\section{REFERENCES}

1. Caselli, P., Myers, P.C., Thaddeus, P. 1995, ApJL, 455, L77 
2. Blatt, S. Ludlow, A.D., Campbell, G.K. et al. 2008, Phys. Rev. Lett. 100, 140801

3. Barrow, J. D., Magueijo, J. 2005 Phys. Rev. D 72, 043521

4. Flambaum, V. V., Kozlov, M. G. 2007, Phys. Rev. Lett. 98, 240801

5. Henkel, C., Menten, K. M., Murphy, M.T. et al. 2009 arXiv:0904.3081

6. Keto, E., and Caselli, P. 2008, ApJ, 683, 238

7. King, J., Webb, J. K., , Murphy, M., T., Carswell, R. F. 2008, Phys. Rev. Lett. 101, 251304

8. Khoury, J., Weltman, A. 2004, Phys. Rev. Lett. 93, 171104

9. Kukolich, S.G. 1967, Phys. Rev. 156, 83

10. Levshakov, S. A., Molaro, P., Kozlov, M. G. 2008, arXiv:0808.0583

11. Molaro, P. Reimers, D., Agafonova, I., Levshakov, S. A. 2008, EPJST, 163, 173

12. Mota, D. F., Barrow, J. D. 2004, MNRAS 349,291

13. Hougen J. T. 1972, J. Chem. Phys., 57, 4207

14. Murphy, M., T., Webb, J., K., Flambaum, V., V. 2008, MNRAS 384, 1053

15. Olive, K., Pospelov, M. 2008 Phys. Rev. D 77, 043524

16. Pagani, L., Daniel, F., Dubernet, M-L. 2009, A\&A, 494, 719

17. Rehinold, E., Buning, R., Hollestein, U., et al. 2006, Phys. Rev. Lett. 96, 151101

18. Rathborne, J. M., Lada, C. J., Muench, A. A., Alves, J. F., Lombardi, M. 2008, ApJS 174,396

19. Rosenband, T. , Hume D.B., Schmidt, P.O. et al. 2008, Science 319, 1808

20. Rosolowsky, E. W., Pineda, J. E., Foster, J. B., et al. 2008, ApJS 175, 509

21. Sakai, T., Sakai, N., Kamegai, K., et al. 2008 ApJ 678, 1049

22. Srianand, R., Chand, H., Petitjean, P., Aracil, B. 2008, Phys. Rev. Lett. 100, 029902

23. Tafalla, M., Myers, P.C., Caselli, P., Walmsley, C. M., Comito, C. 2002, ApJ 569, 815

24. Tafalla, M., Myers, P.C., Caselli, P., Walmsley, C. M. 2004, A\&A, 416, 191

25. Wendt, M., Reimers, D. 2009, EPJST, 163, 197 (astro-ph/0802.1160) 